\title{
Performance of Underwater Ad-Hoc Networks
}

\author{
Andrej Stefanov and Milica Stojanovic \\ Electrical and Computer Engineering Department \\ Northeastern University \\ E-mail: a.stefanov@neu.edu, millitsa@mit.edu
}

\begin{abstract}
We consider the performance of underwater acoustic ad-hoc networks. We assume a uniform distribution of the nodes over a finite area. The node-to-node channel is modeled through the Ricean fading model. We adopt a communication theoretic approach and study the interdependence of the sustainable number of hops through the network, end-to-end frame error probability, power and bandwidth allocation and operating frequency. We find that the network connectivity, given by the sustainable number of hops, exhibits a bimodal behavior. We present numerical examples that illustrate the results of the analysis.
\end{abstract}

\section{INTRODUCTION}

Recently, there has been an increased interest in underwater wireless communications systems [1]. Motivated by the advances in the physical layer and maturation of underwater acoustic modem technology [2], the research in this area is moving towards the development of underwater networking principles. Paramount to this research is the understanding of the acoustic propagation mechanism [3], [4].

In this paper, we focus on an underwater network of bottom mounted nodes. Therefore, we consider a two-dimensional network model. In underwater acoustic communication systems, the power is subject to high attenuation that depends both on the distance and the frequency of the signal, while the bandwidth is severely limited. Hence, we assume multihop transmission based on nearest neighbor routing, as it offers more promising bandwidth and path loss conditions [3]. We study the behavior of the multihop network by performing a communication theoretic analysis.

The paper is organized as follows. Section II describes the underwater acoustic channel model. Section III describes the ad-hoc network set-up. We adopt a communication theoretic approach [5] and investigate the interdependence between the sustainable number of hops in the network, as an indicator of network connectivity, end-to-end frame error probability, power and bandwidth allocation and operating frequency. The initial study focuses on an idealized scenario when there is no interference in the network. Numerical examples illustrating the results of the analysis are presented in Section IV. Concluding remarks are given in section $\mathrm{V}$.

\section{Underwater Acoustic Channel Model}

Underwater acoustic communication channels are characterized by a path loss that depends not only on the distance between the transmitter and the receiver, as is the case in many other wireless channels, but also on the signal frequency.
The absorption loss increases with frequency, as well as with distance and limits the practically usable bandwidth.

\section{A. Attenuation}

Attenuation, or path loss, that occurs in an underwater acoustic channel over a distance $d$ for a signal of frequency $f$, is given by

$$
A(d, f)=A_{0} d^{\kappa} a(f)^{d}
$$

where $A_{0}$ is a unit-normalizing constant that includes fixed losses, $a(f)$ is the absorption coefficient and $\kappa$ is the spreading factor. In the case of practical spreading $\kappa=1.5$. The absorption coefficient can be expressed empirically, using the Thorps formula which gives $a(f)$ in $\mathrm{dB} / \mathrm{km}$ for $f$ in $\mathrm{kHz}$ as [3]

$$
10 \log a(f)=\frac{0.11 f^{2}}{1+f^{2}}+\frac{44 f^{2}}{4100+f^{2}}+\frac{2.75 f^{2}}{10^{4}}+0.003
$$

This formula is generally valid for frequencies above a few hundred Hz.

\section{B. Noise}

The ambient noise in the ocean can be modeled using four sources: turbulence, shipping, waves and thermal noise. Most of the ambient noise sources can be described by Gaussian statistics and a continuous power spectral density. The following empirical formulae give the power spectral densities of the four noise components in $\mathrm{dB}$ re $\mu \mathrm{Pa}$ per $\mathrm{Hz}$ as a function of frequency in $\mathrm{kHz}[3]$

$$
\begin{aligned}
10 \log N_{\mathrm{t}}(f)= & 17-30 \log f, \\
10 \log N_{\mathrm{s}}(f)= & 40+20(s-0.5)+26 \log f \\
& -60 \log (f+0.03), \\
10 \log N_{\mathrm{w}}(f)= & 50+7.5 \sqrt{w}+20 \log f \\
& -40 \log (f+0.4), \\
10 \log N_{\mathrm{th}}(f)= & -15+20 \log f
\end{aligned}
$$

where $s$ is the shipping activity factor, $0 \leq s \leq 1$, and $w$ is the wind speed in $\mathrm{m} / \mathrm{s}$. The overall power spectral density of the ambient noise is

$$
N(f)=N_{\mathrm{t}}(f)+N_{\mathrm{s}}(f)+N_{\mathrm{w}}(f)+N_{\mathrm{th}}(f) .
$$




\section{AN Product and Signal-to-Noise Ratio}

Using the attenuation $A(d, f)$ and the noise power spectral density $N(f)$ we can evaluate the signal-to-noise ratio observed over a distance $d$. The narrow-band signal-to-noise ratio is given by [3]

$$
\operatorname{snr}(d, f)=\frac{S(f)}{A(d, f) N(f)}
$$

where $S(f)$ is the power spectral density of the transmitted signal. The AN product $A(d, f) N(f)$, determines the frequency dependent part of the signal-to-noise ratio. The factor $\frac{1}{A(d, f) N(f)}$ is illustrated in Figure 1. For each transmission distance $d$, there clearly exists a frequency $f_{o}(d)$ for which the narrow-band signal-to noise ratio is maximized. In practice, one may choose $f_{o}(d)$ as the operating frequency and allocate a certain transmission bandwidth around it.

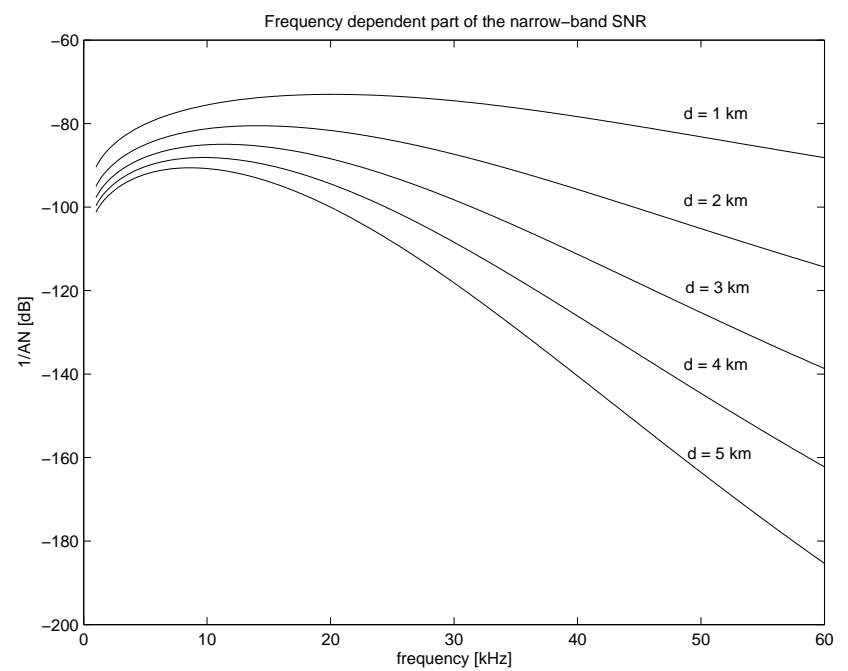

Fig. 1. The AN product for various distances. Spreading factor $\kappa=1.5$.

\section{3 dB Bandwidth}

We define the $3 \mathrm{~dB}$ bandwidth, $B_{3}(d)$, as the range of frequencies around $f_{o}(d)$ for which $A(d, f) N(f)<2 A\left(d, f_{o}(d)\right) N\left(f_{o}(d)\right)$. The operating frequency $f_{o}(d)$ and the $3 \mathrm{~dB}$ bandwidth, $B_{3}(d)$, are presented in Figure 2.

\section{AD-HOC NeTwORK SETUP}

We consider a two dimensional network of bottom mounted nodes that provides coverage over a certain area. We assume a uniform distribution of the nodes as depicted in Figure 3. Given the number of nodes in the network, $N$, and the area of the network, $\mathcal{A}$, the density of the network, $\rho_{s}$, is

$$
\rho_{s}=\frac{N}{\mathcal{A}} \text {. }
$$

Given the uniform node distribution and circular area of the network, the distance between nodes, $d$, is

$$
d=\frac{c}{\sqrt{\rho_{s}}}
$$

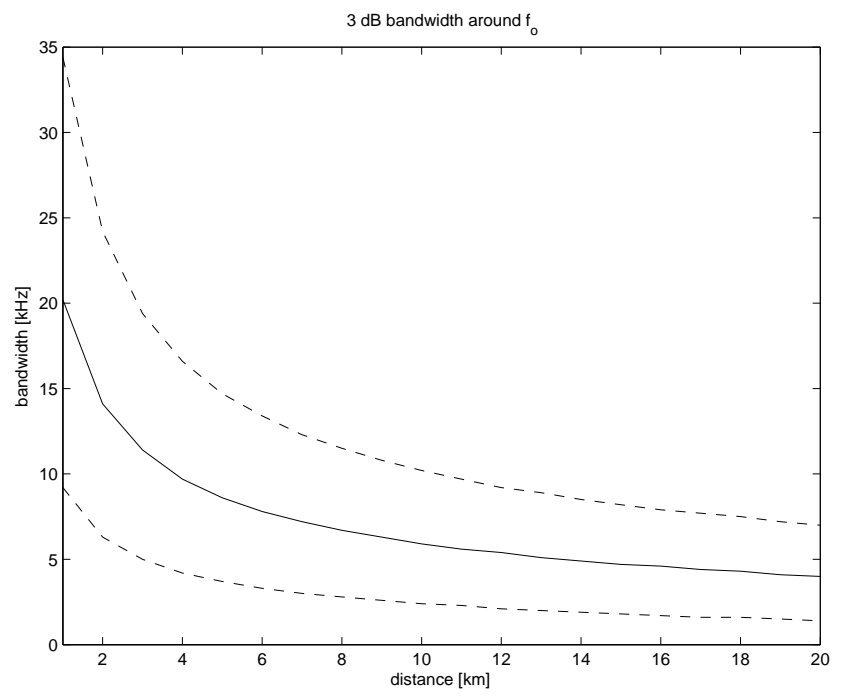

Fig. 2. Frequency $f_{o}(d), f_{\max }(d)$ and $f_{\min }(d)$ which define the $3 \mathrm{~dB}$ bandwidth. Spreading factor $\kappa=1.5$.

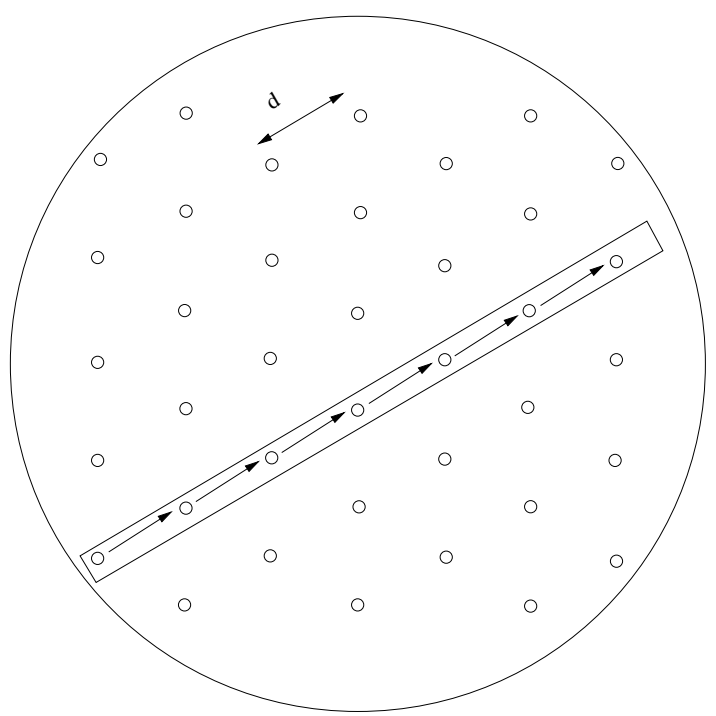

Fig. 3. Uniform network setup.

where $c$ is a constant that depends on the node placement (grid pattern). Without loss of generality we assume that $c=1$.

We assume multihop transmission based on nearest neighbor routing. This is an energy saving strategy that is attractive for networks with battery powered nodes. As the longest multihop route in the network is along the diameter of the network, $D$, the maximum number of hops, $n_{h}^{\max }$ is

$$
n_{h}^{\max }=\frac{D}{d}=\frac{2 \sqrt{\mathcal{A} / \pi}}{\sqrt{\mathcal{A} / N}}=\frac{2}{\sqrt{\pi}} \sqrt{N} .
$$

Let the average number of hops for a multihop route be $\bar{n}_{h}$. Then, as long as the probability distribution of the number of hops is symmetric, we have [5]

$$
\bar{n}_{h}=\frac{n_{h}^{\max }}{2}=\sqrt{\frac{N}{\pi}} .
$$


We assume uncoded BPSK transmission with a simple demodulate and forward strategy employed by the relays. The end-to-end frame error probability for a multihop route with $n_{h}$ hops, $p_{\text {route, }}$ is given by

$$
p_{\text {route }}=1-\left(1-p_{b}\right)^{L n_{h}}
$$

where $p_{b}$ denotes the bit error probability of a single node-tonode link and $L$ denotes the frame size in bits.

We consider the quality-of-service for the network in terms of the maximum allowed end-to-end route frame error probability, i.e., we require $p_{\text {route }} \leq p_{\text {route }}^{\max }$. Let the number of hops that can be sustained by the network, i.e., the number of hops that can satisfy the maximum end-to-end route frame error probability, be denoted by $n_{\mathrm{sh}}$. From Eq. (10) it follows that $n_{\text {sh }}$, can be calculated as ${ }^{1}$

$$
n_{\mathrm{sh}}=\frac{1}{L} \frac{\log \left(1-p_{\text {route }}^{\max }\right)}{\log \left(1-p_{b}\right)} \approx \frac{1}{L} \frac{p_{\text {route }}^{\max }}{p_{b}} .
$$

We focus on a frequency non-selective Ricean fading model for the node-to-node channel [6]. Under the assumption that perfect channel state information is available at the receiver, the bit error probability, $p_{b}$, can be upper bounded as [7]

$$
p_{b} \leq \frac{1+\mathcal{K}}{1+\mathcal{K}+\operatorname{snr}(d, f)} \exp \left(-\frac{\mathcal{K} \operatorname{snr}(d, f)}{1+\mathcal{K}+\operatorname{snr}(\mathrm{d}, \mathrm{f})}\right)
$$

where $\mathcal{K}$ denotes the Ricean factor and the signal-to-noise ratio is calculated for the operating frequency $f_{o}(d)$ as

$$
\mathrm{snr}=\frac{P}{A\left(d, f_{o}\right) N\left(f_{o}\right) B}=\frac{P}{A\left(\sqrt{\frac{\mathcal{A}}{N}}, f_{o}\right) N\left(f_{o}\right) B} .
$$

We note that the frequency-nonselective Ricean fading assumption is a suitable approximation for systems with narrow bandwidth. For systems with wide bandwidth the approximation is valid given that a multi-carrier communication system such as OFDM is utilized [8]. In that scenario, the operating frequency, $f_{o}(d)$, would describe the performance on one of the carriers. The performance on the other carriers would depend on a frequency given by $f_{o}(d) \pm \Delta$.

\section{Numerical Results}

We present numerical examples that illustrate the performance of underwater acoustic ad-hoc networks. We examine the relationships between the sustainable number of hops, the end-to-end frame error probability, the signal power, bandwidth, and the operating frequency. We assume independent Ricean fading for each node-to-node channel with $\mathcal{K}=10$. We consider a target (maximum allowed) end-to-end frame error probability of $p_{\text {route }}=10^{-3}$. We assume circular network of area $\mathcal{A}=1000 \mathrm{~km}^{2}$. We note that an acoustic signal propagates as a pressure wave whose level is commonly measured in $\mathrm{dB}$ relative to $1 \mu \mathrm{Pa}$. We adapt that convention, hence the power levels are expressed in $\mathrm{dB}$ re $\mu \mathrm{Pa}$. We neglect

\footnotetext{
${ }^{1}$ Note that while the analysis does not consider it explicitly, in practice $\left\{n_{\mathrm{sh}}, n_{h}^{\max }\right\} \in \mathbb{N}$.
}

any fixed losses. ${ }^{2}$ The frame size $L=100$ bits, the spreading factor $\kappa=1.5$, the shipping activity factor $\mathrm{s}=0.5$ and we assume calm conditions, that is, the wind speed $w=0 \mathrm{~m} / \mathrm{s}$.

Figure 4 presents the sustainable number of hops for an endto-end frame error probability of $\mathrm{FEP}_{\text {route }}=10^{-3}$, bandwidth $B=4 \mathrm{kHz}$ and transmit power $P=115 \mathrm{~dB}$ re $\mu \mathrm{Pa}$. The average number of hops given by Eq. (9) is also presented. We observe that the sustainable number of hops in the network exhibits a bimodal behavior. Below about $N=300$ nodes, the network can not sustain routes with an average number of hops. This is due to the fact that with fewer than 300 nodes in the network, the nodes are too far apart to guarantee the required end-to-end frame error probability for the available transmit power. As the number of nodes increases above 300 nodes, we see that the sustainable number of hops rapidly exceeds the average number of hops. When the number of nodes is $N \gtrsim 400$, we have $n_{\text {sh }} \geq n_{h}^{\max }$. This ensures full connectivity in the network, where all routes satisfy the endto-end frame error probability requirement. The operating frequency $f_{o}(d)$ and the node-to-node signal-to-noise ratio are also presented. The operating frequency increases with $N$ as the node-to-node distance decreases. The signal-to-noise ratio changes for fixed transmit power $P$, because the AN product changes with the distance and the frequency.
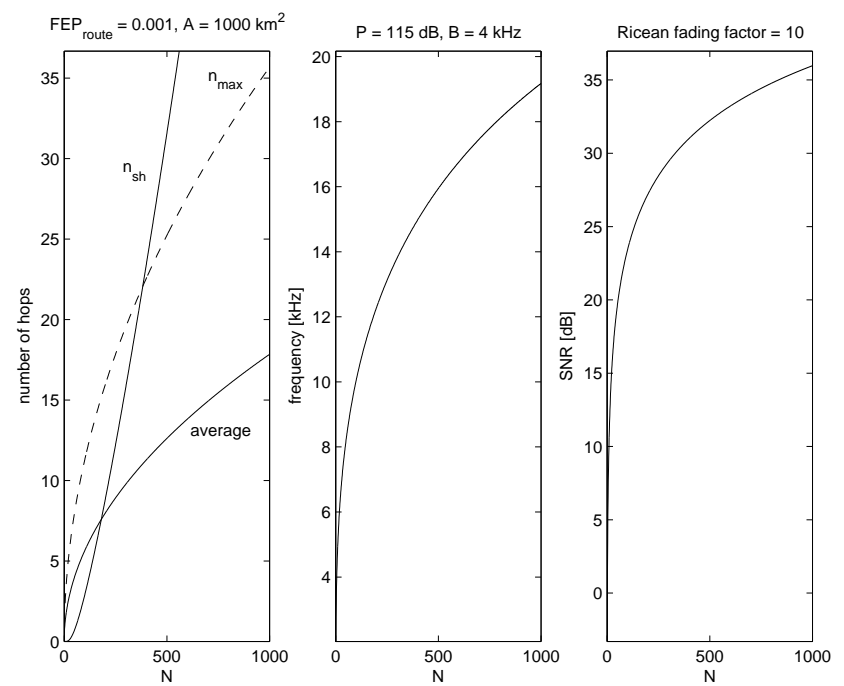

Fig. 4. Sustainable number of hops, operating frequency and SNR for a uniform network with Ricean fading. The area $\mathcal{A}=1000 \mathrm{~km}^{2}$, bandwidth $B=4 \mathrm{kHz}$, transmit power $P=115 \mathrm{~dB}$ re $\mu \mathrm{Pa}$.

Figure 5 depicts the sustainable number of hops for different values of the bandwidth. The end-to-end frame error probability is $\mathrm{FEP}_{\text {route }}=10^{-3}$ and the transmit power is $P=115 \mathrm{~dB}$ re $\mu \mathrm{Pa}$. We observe that the sustainable number of hops decreases as we increase the signal bandwidth. For example, bandwidths of $B=1 \mathrm{kHz}$ and $B=4 \mathrm{kHz}$ result

\footnotetext{
${ }^{2}$ Inclusion of additional frequency independent losses, and an adjustment of the background noise level to suit a particular environement and provide the necessary SNR margins, will scale the results in absolute value, but will not alter the general behavior.
} 
in full connectivity when the number of nodes $N \gtrsim 50$ and $N \gtrsim 375$, respectively. However, if the bandwidth is $B=10 \mathrm{kHz}$, full connectivity can not be achieved. When the number of nodes $N \lesssim 550$ even routes with an average number of hops can not be sustained. This is due to the increase in the noise power, as the bandwidth increases.

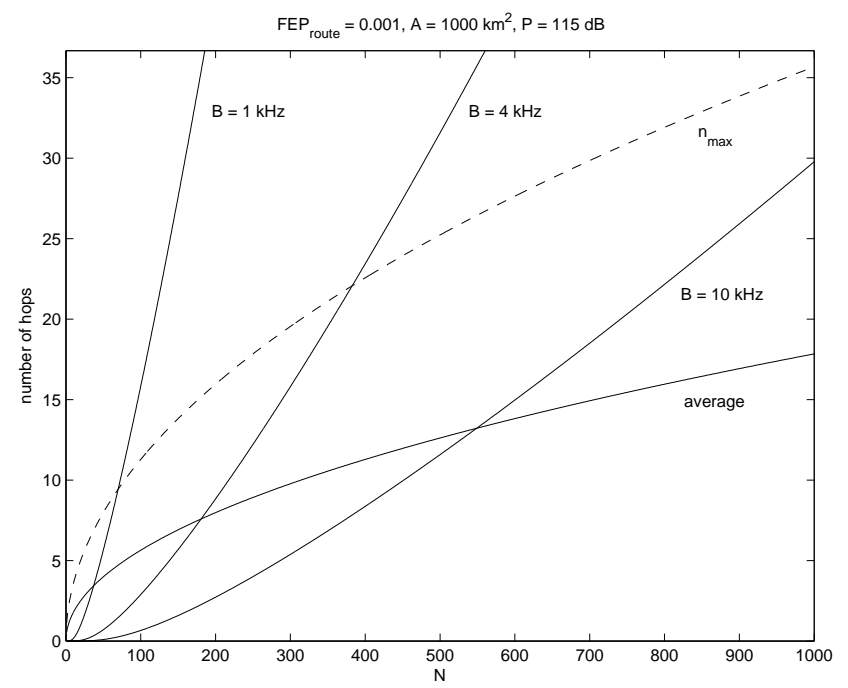

Fig. 5. Sustainable number of node-to-node hops for a uniform network with Ricean fading with varying signal bandwidth. The area $\mathcal{A}=1000 \mathrm{~km}^{2}$, transmit power $P=115 \mathrm{~dB}$ re $\mu \mathrm{Pa}$.

Figure 6 presents the sustainable number of hops for different values of the transmit power. The end-to-end frame error probability is $\mathrm{FEP}_{\text {route }}=10^{-3}$ and the bandwidth is $B=4 \mathrm{kHz}$. We observe that the sustainable number of hops decreases as we decrease the signal power. For example, transmit powers of $115 \mathrm{~dB}$ re $\mu \mathrm{Pa}$ and $120 \mathrm{~dB}$ re $\mu \mathrm{Pa}$ result in full connectivity when the number of nodes in the network $N \gtrsim 80$ and $N \gtrsim 350$, respectively. However, if the transmit power is reduced to $P=110 \mathrm{~dB}$ re $\mu \mathrm{Pa}$, full connectivity can not be achieved. When the number of nodes $N \lesssim 700$ even routes with an average number of hops can not be sustained.

The sensitivity of the sustainable number of hops to the carrier frequency is presented in Figure 7. The end-to-end frame error probability is $\mathrm{FEP}_{\text {route }}=10^{-3}$, the bandwidth is $B=4 \mathrm{kHz}$ and the transmit power is $P=120 \mathrm{~dB}$ re $\mu \mathrm{Pa}$. We observe that the choice of $f_{o}+4 \mathrm{kHz}$ instead of $f_{o}$ as the carrier frequency results in a very similar performance with respect to the sustainable number of hops. As can be seen in Figure 8, the signal-to-noise ratio at $f_{o}+4 \mathrm{kHz}$ is also very close to the signal-to-noise ratio achieved at $f_{o}$. Even the choice of $f_{o}+8 \mathrm{kHz}$ or $f_{o}+12 \mathrm{kHz}$ instead of $f_{o}$, would result in a requirement of around only 50 or 150 additional nodes to achieve the average number of hops through the network. We also note that similar performance would be observed if the chosen frequencies were of the form $f_{o}-\Delta$ rather than $f_{o}+\Delta$.

The sustainable number of hops in the case of $3 \mathrm{~dB}$ bandwidth allocation around $f_{o}$ is presented in Figure 9.

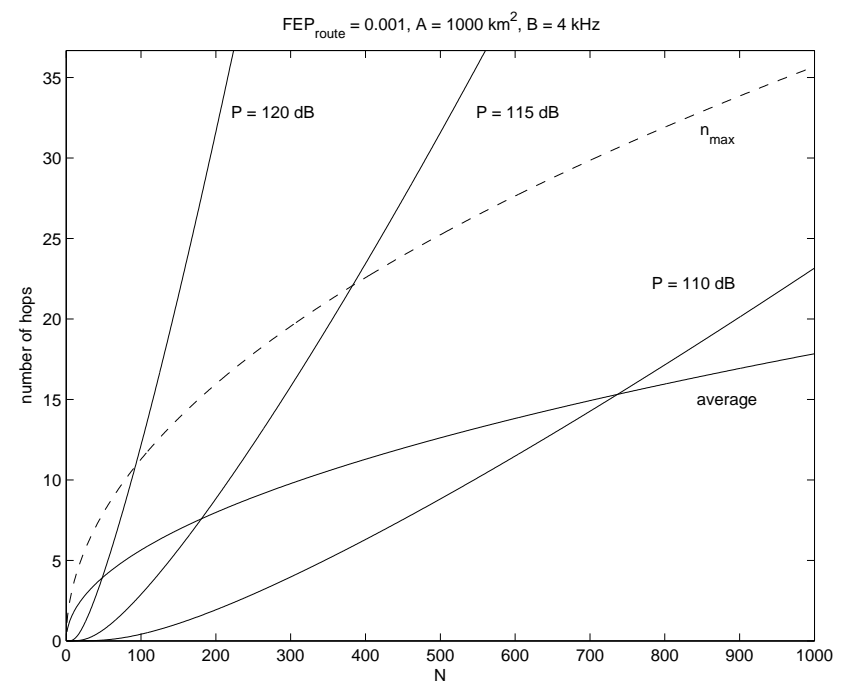

Fig. 6. Sustainable number of node-to-node hops for a uniform network with Ricean fading with varying signal power. The area $\mathcal{A}=1000 \mathrm{~km}^{2}$, bandwidth $B=4 \mathrm{kHz}$.

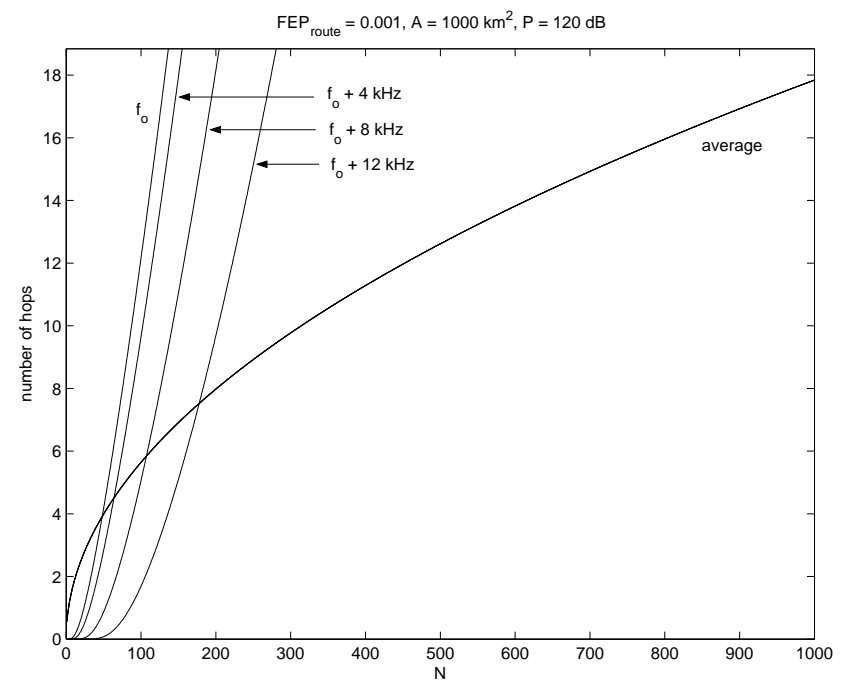

Fig. 7. Sustainable number of node-to-node hops for uniform network with Ricean fading vs. the average number of hops for various carrier frequencies: $f_{o} \mathrm{kHz}, f_{o}+4 \mathrm{kHz}, f_{o}+8 \mathrm{kHz}$ and $f_{o}+12 \mathrm{kHz}$. The area $\mathcal{A}=1000 \mathrm{~km}^{2}$, bandwidth $B=4 \mathrm{kHz}$, transmit power $P=120 \mathrm{~dB}$ re $\mu \mathrm{Pa}$.

The end-to-end frame error probability is $\mathrm{FEP}_{\text {route }}=10^{-3}$ and we assume constant transmit power spectral density of $110 \mathrm{~dB}$ re $\mu \mathrm{Pa}$ per $\mathrm{Hz}$. We also present $f_{o}$ and the $3 \mathrm{~dB}$ bandwidth. We observe that the $3 \mathrm{~dB}$ bandwidth increases as the number of nodes in the network increases. This is due to the fact that the distance between nodes in the network decreases as $N$ increases, and as depicted in Figure 1, as the distance decreases, $\frac{1}{A(d, f) N(f)}$, increases and becomes smoother.

Figure 10 presents the sustainable number of hops in the scenario where the nodes have the possibility of adjusting their power. The end-to-end frame error probability is $\mathrm{FEP}_{\text {route }}=10^{-3}$ and the bandwidth is $B=4 \mathrm{kHz}$. We still 


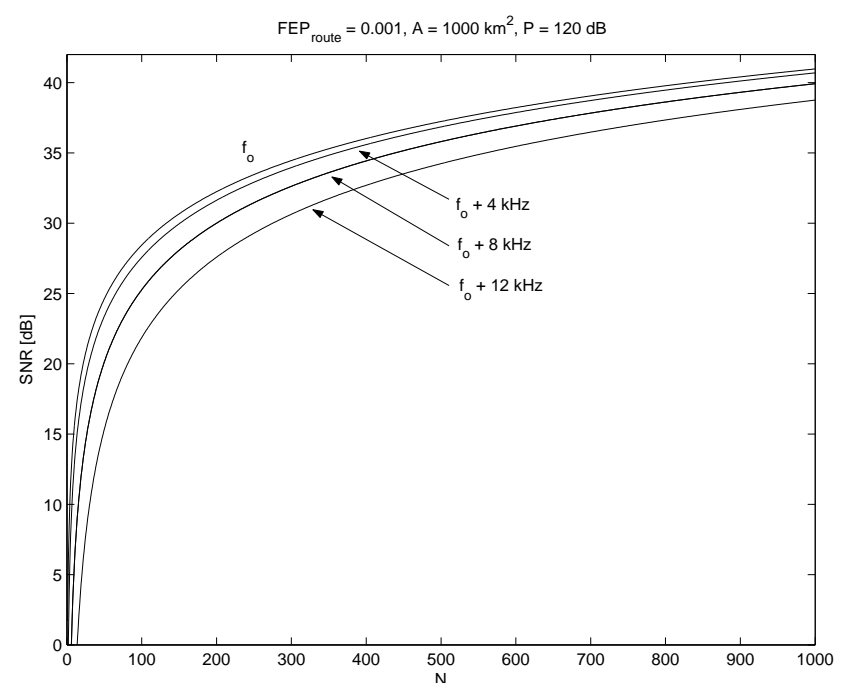

Fig. 8. Signal-to-noise ratios per hop for operating frequency of $f_{o} \mathrm{kHz}$, $f_{o}+4 \mathrm{kHz}, f_{o}+8 \mathrm{kHz}$ and $f_{o}+12 \mathrm{kHz}$. The area $\mathcal{A}=1000 \mathrm{~km}^{2}$, bandwidth $B=4 \mathrm{kHz}$, transmit power $P=120 \mathrm{~dB}$ re $\mu \mathrm{Pa}$.
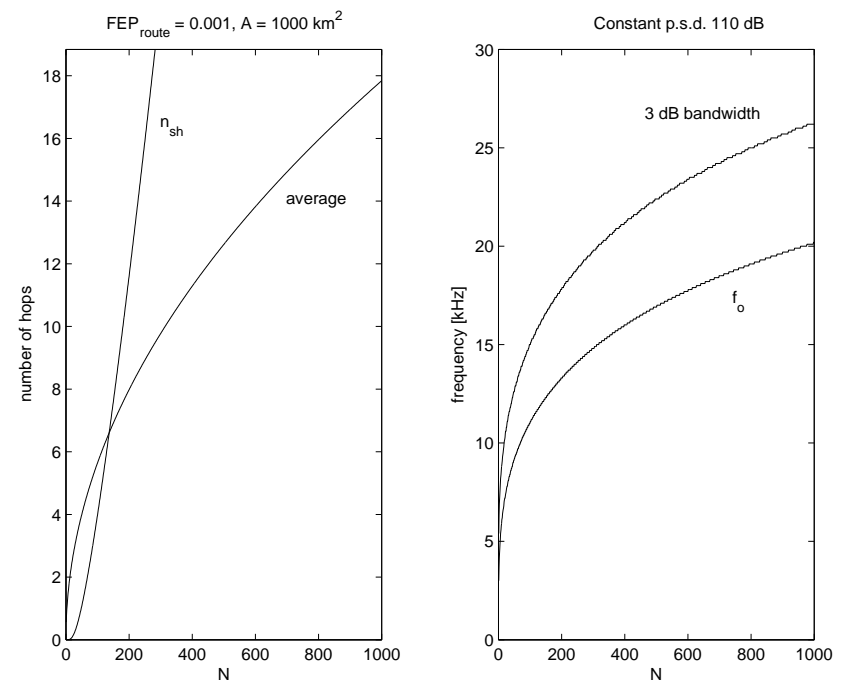

Fig. 9. Sustainable number of node-to-node hops for uniform network with Ricean fading. The area $\mathcal{A}=1000 \mathrm{~km}^{2}, 3 \mathrm{~dB}$ bandwidth $B_{3}(d)$, constant power spectral density $P / B_{3}(d)=110 \mathrm{~dB}$ re $\mu$ Pa per Hz.

observe the bimodal behavior of the sustainable number of hops, however the sustainable number of hops is constrained so that $n_{\mathrm{sh}} \leq n_{h}^{\max }$. That is, the number of hops through the network is constrained by Eq. (8), the maximum number of hops given the total number of nodes in the network, $N$. Hence, as the number of nodes in the network increases and the distance between nodes decreases, it is possible to reduce the transmit power. For example, when the number of nodes in the network $N=1000$, a transmit power of around $112 \mathrm{~dB}$ re $\mu \mathrm{Pa}$ is sufficient to achieve the required end-toend frame error probability of $\mathrm{FEP}_{\text {route }}=10^{-3}$, resulting in savings of around $8 \mathrm{~dB}$ re $\mu \mathrm{Pa}$ compared to the constant power scenario.
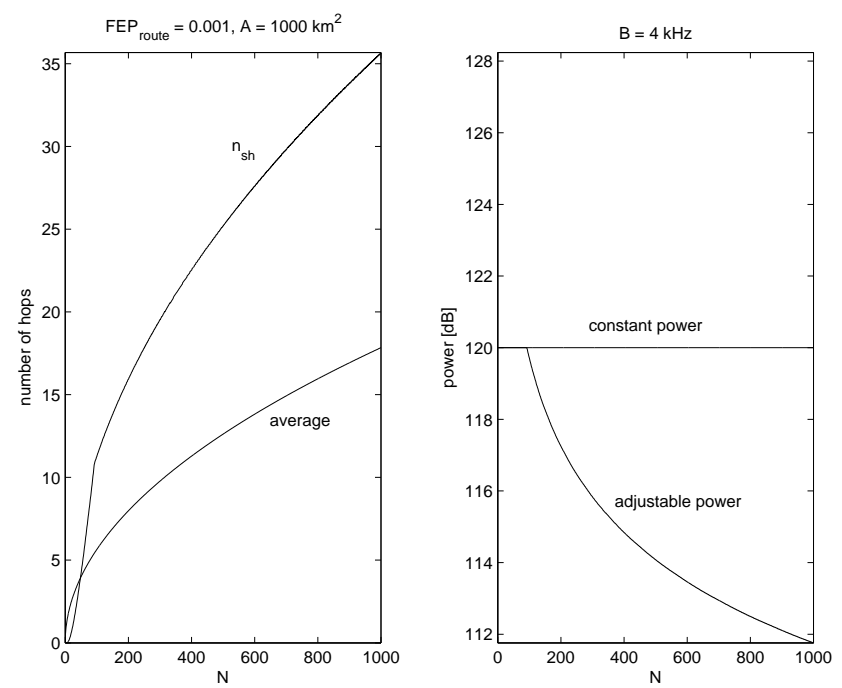

Fig. 10. Sustainable number of node-to-node hops for a uniform network with Ricean fading with adjustable power nodes. The area $\mathcal{A}=1000 \mathrm{~km}^{2}$, bandwidth $B=4 \mathrm{kHz}$.

\section{CONCLUSions}

We investigated the performance of underwater acoustic ad-hoc networks in an idealized scenario when there is no interference in the network. In particular, we adopted a communication theoretic approach and studied the interdependence between the sustainable number of hops in the network, end-to-end frame error probability, power and bandwidth allocation, and operating frequency. We observed that the network connectivity, described by the sustainable number of hops, exhibited a bimodal behavior. Numerical examples were presented to illustrate the results of the analysis.

\section{ACKNOWLEDGMENTS}

This work was supported in part by the NSF grant 0831728 and the ONR grant N00014-09-1-0700.

\section{REFERENCES}

[1] IEEE Journal on Selected Areas in Communications, Special Issue on Underwater Wireless Communications and Networks, December 2008.

[2] L. Freitag et al., "The WHOI Micro-Modem: An Acoustic Communications and Navigation System for Multiple Platforms," in Proc. IEEE Oceans Conference, 2005.

[3] M. Stojanovic and J. Preisig, "Underwater Acoustic Communication Channels: Propagation Models and Statistical Characterization," IEEE Communications Magazine, pp. 84-89, January 2009.

[4] M. Stojanovic, "On the Relationship Between Capacity and Distance in an Underwater Acoustic Channel," ACM SIGMOBILE Mobile Comp. Commun. Rev., vol. 11, No. 4, pp. 34-43, October 2007.

[5] O. Tonguz and G. Ferrari, "Ad Hoc Wireless Networks: A Communication-Theoretic Perspective," Wiley, 2006.

[6] P. Qarabaqi and M. Stojanovic, "Statistical Modeling of a Shallow Water Acoustic Communication Channel," in Proc. Underwater Acoustic Measurements Conference, Nafplion, Greece, June, 2009.

[7] S. Benedetto and E. Biglieri, "Principles of Digital Transmission with Wireless Applications," Kluwer/Plenum, 1999.

[8] L. Hanzo and T. Keller, "OFDM and MC-CDMA: A Primer," WileyIEEE Press, 2006. 